\section{Adding mirabegron to solifenacin improves patient outcomes}

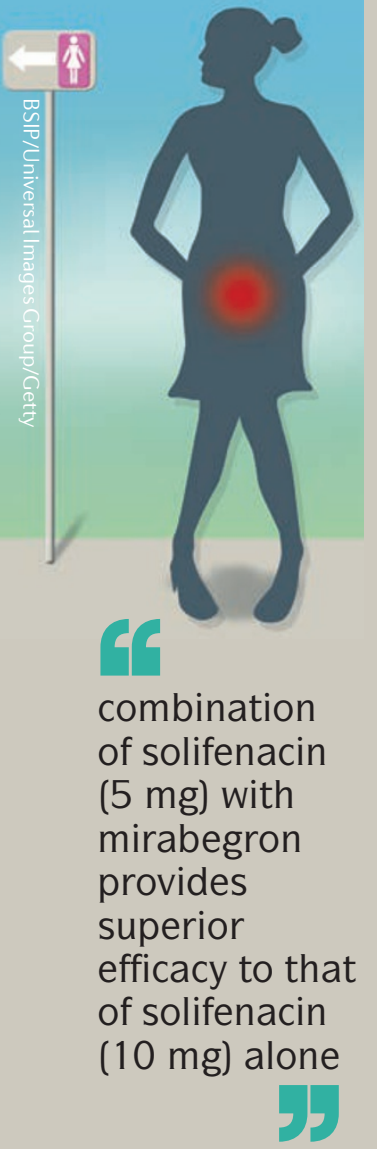

Antimuscarinics have remained the standard of care for patients with overactive bladder (OAB) syndrome for several decades, despite the fact that, owing to their adverse effects, these drugs are poorly tolerated by most patients. Now, data from the BESIDE study, a randomized phase III trial comparing mirabegron plus solifenacin with solifenacin alone, reveal significant improvements in terms of both urinary incontinence and self-reported quality of life outcomes in patients receiving solifenacin plus mirabegron.

2,174 patients with an inadequate response to solifenacin ( $5 \mathrm{mg}$ ) monotherapy for a minimum of 4 weeks were randomized in a 1:1:1 ratio to receive daily oral treatment with either $5 \mathrm{mg}$ solifenacin, $10 \mathrm{mg}$ solifenacin or $5 \mathrm{mg}$ solifencin plus mirabegron (25-50 mg). Patients' symptoms were monitored for 12 weeks. Recognizing the importance of patient preferences, patient-reported outcomes were measured using a total of seven different responder analyses including those based on efficacy outcomes, health-related quality of life (HRQoL) and patient perception of bladder condition. After 12 weeks of treatment, patients in all treatment groups had a reduction in the mean number of incontinence episodes per 24 hours, which was the primary study end point; however, patients who received mirabegron plus solifenacin had a significantly greater reduction than those receiving either $5 \mathrm{mg}$ solifenacin or $10 \mathrm{mg}$ solifenacin alone. Similar changes were observed in other urodynamic parameters including a significant reduction in the mean number of micturitions per 24 hours in the solifenacin plus mirabegron group and a significant increase in the mean volume voided per micturition in this treatment group. No significant differences were observed in terms of changes in nocturia episodes across different treatment groups.

Similar to the clinical outcomes, patients in all treatment groups had improvements in their self-reported HRQoL scores compared with baseline; however, patients in the solifenacin plus mirabegron group again had significantly greater improvements in $\mathrm{HRQ}$ oL scores than those receiving either dose of solifenacin monotherapy.

Despite these apparent

improvements in self-reported HRQoL scores in patients receiving solifencain plus mirabegron, these findings were not entirely reflected by the frequencies of treatment-emergent adverse events (TEAEs) across the three groups. $35.9 \%$ of patients in the combination group had at least one TEAE compared with $33.1 \%$ in the solifenacin $5 \mathrm{mg}$ group, and these included slightly increased risks of the most common adverse effects of antimuscarinics: dry mouth and constipation. The significant improvement in self-reported HRQoL scores reported by patients receiving tolerodine plus mirabegron would imply either that these differences in risk of TEAEs are insignificant, or that patients are willing to accept more adverse events in return for a more effective treatment.

These data confirm that the combination of solifenacin $(5 \mathrm{mg})$ with mirabegron provides superior efficacy to that of solifenacin $(10 \mathrm{mg})$ alone, with a similar frequency of TEAEs and greater self-reported HRQoL scores. Comparisons of solifenacin with mirabegron alone remain largely lacking.

Peter Sidaway

ORIGINAL ARTICLES Drake, M. J. et al. Efficacy and safety of mirabegron add-on therapy to solifenacin in incontinent overactive bladder patients with an inadequate response to initial 4-week solifenacin monotherapy: a randomised double-blind multicentre phase 3B study (BESIDE). Eur. Urol. http://dx.doi.org/10.1016/j.

eururo.2016.02.030 (2016) | MacDiarmid, S. et al. Mirabegron as add-on treatment to solifenacin in incontinent overactive bladder patients with an inadequate response to solifenacin monotherapy: responder analyses and patient-reported outcomes from the BESIDE study. J. Urol. http:// dx.doi.org/10.1016/j.juro.2016.03.174 (2016) 